\title{
Iron therapy substantially restores qEEG maturational lag among iron-deficient anemic infants
}

\section{Gloria A. Otero, Thalía Fernández, F. Bernardo Pliego-Rivero \& Gustavo G. Mendieta}

To cite this article: Gloria A. Otero, Thalía Fernández, F. Bernardo Pliego-Rivero \& Gustavo G. Mendieta (2017): Iron therapy substantially restores qEEG maturational lag among iron-deficient anemic infants, Nutritional Neuroscience, DOI: 10.1080/1028415X.2017.1391529

To link to this article: http://dx.doi.org/10.1080/1028415X.2017.1391529

\section{曲 Published online: 24 Oct 2017.}

Submit your article to this journal $[\pi$

a)

View related articles $\widetilde{ }$

View Crossmark data $\nearrow$ 


\title{
Iron therapy substantially restores qEEG maturational lag among iron-deficient anemic infants
}

\section{Gloria A. Otero $\oplus^{1}$, Thalía Fernández $\odot^{2}$, F. Bernardo Pliego-Rivero $\oplus^{1}$, Gustavo G. Mendieta ${ }^{3}$}

\author{
${ }^{1}$ Facultad de Medicina, Universidad Autónoma del Estado de México, Toluca, México, ${ }^{2}$ Instituto de \\ Neurobiología, Universidad Nacional Autónoma de México, Campus Juriquilla, Querétaro, México, ${ }^{3} \mathrm{Hospital}$ \\ para el Niño, IMIEM, Toluca, México
}

\begin{abstract}
Objective: To use quantitative electroencephalography ( $q E E G$ ) to assess the impact of iron-deficiency anemia on central nervous system maturation in the first year of life.

Method: Twenty-five infants (3-12 months old) presenting ferropenic anemia (IDA) and 25 healthy controls (CTL1), matched by age/gender with the former, were studied in two stages. Electroencephalogram during spontaneous sleep was recorded from all participants; the fast Fourier transform was calculated to obtain absolute power (AP) and relative power (RP) qEEG measures. In the first stage, a qEEG comparison between CTL1 and IDA was performed. Second stage consisted in comparing aEEG of the IDA infants before and after supplementation with iron (IDA-IS group), and comparing qEEG of the IDA-IS group with another control age-matched group (CTL2). Non-parametric multivariate permutation tests (NPT) were applied to assess differences between CTL1 and IDA groups, as well as IDA vs. IDA-IS, and IDA-IS vs. CTL2.

Results: More power in slow frequency bands and less power in fast frequency bands in 64\% of IDA babies were observed. NPT evinced higher alpha AP and RP $(P<0.001)$, less theta AP, and less delta and theta RP in CTL1 than in IDA. After iron-restoration therapy, alpha AP and RP increased while theta AP and theta and delta RP decreased, reaching almost normal values.

Discussion: This work reveals CNS developmental delay through the study of qEEG (less rapid and more slow frequencies) which recovered significantly with iron supplementation. It is concluded that IDA constitutes a high risk factor for a lag of CNS maturation.
\end{abstract}

Keywords: QEEG, Frequency analysis of EEG, Infants, Iron-deficiency anemia, qEEG underdevelopment

\section{Introduction}

Dietary iron deficiency persists as the most frequent dietary shortage worldwide, even though its treatment has been known for decades. ${ }^{1,2}$ While the prevalence of anemia is estimated at $9 \%$ in highly developed countries, in underdeveloped countries, it is reported to be of $43 \%{ }^{3,4}$ During lactation, iron requirements of the baby are fulfilled by maternal milk. ${ }^{5}$ Nevertheless, bodily needs rise markedly 4-6 months after birth and up to the first year of age. It has been calculated that iron requirements during this period range from 0.7 to $0.9 \mathrm{mg} /$ day. ${ }^{6}$ An important research effort has been made to determine the impact of IDA on the psychomotor and cognitive development of afflicted infants. Infants suffering from iron-deficiency

Correspondence to: Gloria A. Otero, Facultad de Medicina, Universidad Autónoma del Estado de México, Paseo Tollocan s/n, col Moderna de la Cruz, Toluca 50180, México. Email: oeog45@gmail.com anemia (IDA) perform poorly in psychomotor development tests, ${ }^{2,7-9}$ which has been corroborated by other studies. ${ }^{10,11}$ However, few attempts have been made to assess CNS maturation employing neurophysiological techniques in IDA infants. For instance, myelination has been assessed through visual and brainstem auditory evoked potentials. ${ }^{12,13}$ Burden et al. ${ }^{14}$ recorded event-related potential in a group of IDA and a group of iron-sufficient toddlers ( 9 and/ or 12 months) during a test of the infant's ability to discriminate a highly familiar stimulus, the mother's face, from a stranger's face. A negative wave associated with attention and a positive slow wave associated with memory updating were identified at both ages both groups. At the age of 9 months, the iron-sufficient group showed a greater negative component, (attentional response) to the mother and a greater updating of memory for the stranger (positive slow wave). This 
pattern appeared until 12 months of age in IDA babies. These authors concluded that iron-deficient infants lacked an appropriate attentional response and exhibited lower updating of memory compared to their iron-sufficient peers. They considered that IDA adversely affects the allocation of neurophysiological resources to the processes of attention and recognition memory during the processing of information of the task. This delay in cognitive development may reflect alterations in the efficiency of central nervous system functions that could be related to the early iron deficiency. Correspondingly, Monga et al. ${ }^{15}$ studied flash visual evoked potentials (VEP) in 25 IDA and 25 healthy babies between the ages of 6 and 24 months. VEP waves N1, P1 and N2, showed longer latencies in the anemic group compared with the control group. Also, a negative correlation was found between the severity of iron-deficiency anemia and latencies of waves of VEP. According to the authors, the mentioned alteration could explain the differences observed in IDA children in psychomotor development.

Considering that the relationship 'iron-cognition' has been extensively investigated using a wide range of behavioral tests, we evaluated the impact of IDA on CNS development from a neurobiological perspective applying EEG frequency analysis (quantitative electroencephalography, qEEG). To postulate our hypothesis, we considered that (a) iron is required for myelination and dendritic arborization; ${ }^{16,17}$ (b) the synthesis and reuptake of central neurotransmitters is negatively affected under IDA; ${ }^{18,19}$ and (c) EEG fundamentally results from cortical synaptic activity. ${ }^{20}$ Therefore, we hypothesized that (1) in relation to their peers without the metal deficiency infants with IDA will reveal an immature EEG power spectrum, i.e. the persistence of absolute and relative potencies in the slow frequency range (delta and theta) and a lack of alpha frequency, (2) iron supplementation would improve the qEEG's maturational features.

Table 1 Hematological data

\begin{tabular}{|c|c|c|c|c|c|c|}
\hline & \multicolumn{2}{|c|}{ CTL } & \multicolumn{2}{|c|}{ IDA } & \multicolumn{2}{|c|}{ IDA-IS } \\
\hline & Mean & SD & Mean & SD & Mean & SD \\
\hline $\mathrm{Hb}(\mathrm{g} / \mathrm{dl})$ & 13.4 & 1.14 & $8.2^{*}$ & 0.83 & $12.4^{\dagger}$ & 1.15 \\
\hline $\mathrm{RC}\left(\times 10^{6}\right)$ & 4.7 & 0.46 & $2.9^{*}$ & 0.19 & $4.2^{\dagger}$ & 0.50 \\
\hline $\mathrm{Htc}(\%)$ & 36.7 & 1.2 & $29.4^{*}$ & 1.70 & $34.5^{\dagger}$ & 0.48 \\
\hline $\operatorname{MCV}\left(\mu m^{3}\right)$ & 99.2 & 1.9 & $61.0^{\star}$ & 2.34 & $94.8^{\dagger}$ & 2.43 \\
\hline $\mathrm{Fe}(\mu \mathrm{g} / \mathrm{dl})$ & 105.8 & 8.40 & $36.8^{*}$ & 5.76 & $97.4^{\dagger}$ & 8.20 \\
\hline Ferr $(\mu \mathrm{g} / \mathrm{dl})$ & 130.2 & 3.70 & $21.6^{\star}$ & 3.02 & $92.4^{\dagger}$ & 7.02 \\
\hline Sat-Trans (\%) & 42.2 & 1.7 & $28.6^{*}$ & 1.30 & $38.0^{\dagger}$ & 1.31 \\
\hline
\end{tabular}

$\mathrm{Hb}$ : hemoglobin; RC: red cells; Htc: hematocrit; MCV: mean corpuscular volume; Fe: serum iron; Ferr: ferritin; Sat-Trans: transferrin saturation.

Significant differences:

${ }^{*}$ CTL vs. IDA.

${ }^{\dagger}$ IDA-S vs. IDA.

\section{Methods}

Study design: Prospective observational study.

\section{Participants}

This research project was pre-approved by the Bioethics Committee of the School of Medicine, UAEMEX and by the participating hospitals. The nature of the study, its aims, and procedures were explained verbally to the children's parents. This explanation included the possible benefits, discomforts and/or risks posed to the child by the test. It was also clarified that the study was confidential, that they could if they wished to abandon the study at any moment, and that the results of the study were going to be delivered to them completely free of any charge. All information was included in the written consent form that was signed by agreeing parents before enrollment.

The sample consisted of 50 children between 3 and 12 months of age. Twenty-five IDA infants were gathered from the external practice of two public state hospitals in Toluca City, State of Mexico, where infants were diagnosed as anemic. The hospitals were: Children's Hospital, IMIEM and General Hospital Adolfo López Mateos, ISEM. The criterion established to consider a status of anemia was hemoglobin $(\mathrm{Hb})$ content under $11 \mathrm{~g} / \mathrm{dl}^{21}$ The ferropenic anemia was verified by the iron's profile (see Table 1). Considering that Toluca City is located at $2650 \mathrm{~m}$ above sea level, the observed $\mathrm{Hb}$ concentrations in IDA infants were unquestionably low (Table 1). The following other inclusion criteria were considered: single birth and pregnancy; born at full term after eutocic labor; parental lack of significant pathological background, including toxicological history and neurological or metabolic disorders such as diabetes; weight at birth between 2500 and $3900 \mathrm{~g}$; Apgar $\geq 8$ within the first minute, and $\geq 9$ at 5 minutes after birth; normal results from pediatric and neurological clinical examinations; and no other signs of malnutrition in addition to IDA at the moment of this study.

The control group (CTL1) was formed by 25 children, paired by age and gender to the IDA sample. CTL1 infants belonged to a previously studied sample of 151 children whose EEGs were used to create the qEEG norms. ${ }^{22}$ For this study, the EEGs and clinical data of the selected age and gendermatched controls were compared with the newly gathered group of IDA infants. Hence, CTL1 children fulfilled the same inclusion criteria, except for their normality regarding bodily iron content and hematological profile.

\section{Experimental procedures}

The study was carried out in two stages. In the first stage, qEEG variables (described below) from the 
IDA group and CTL1 group of healthy infants were compared. In the second stage two comparisons were made: qEEG variables of the IDA infants before and after four months of iron supplementation were compared, and the qEEG variables of the IDA infants after iron supplementation (IDA-IS group) were also compared with those of a new control group (CTL2), which fulfilled the same inclusion criteria than the CTL1 group and were also paired by age and gender to the IDA-IS sample. Since treatment was given for 4 months, infants who started with 12 months ended with 16 months, but the norm mentioned covers up to 12 months, it was necessary to capture new control children between 12 and 16 months, on the other hand, those who began the therapy at 3 months of age were 7 months old when they finished, so at the second phase of the study the sample age range was 7-16 months, so it was necessary to complete the CTL group of the second phase in order to complete 25 infants.

EEG recordings were obtained from all participants, and they were performed in a dim lit and soundproof room during spontaneous sleep. While recording, all children remained on the lap of their mothers and wore a polyester cap with surface electrodes distributed according to the 10-20 International System (Fp1, Fp2, F3, F4, C3, C4, P3, P4, O1, O2, F7, F8, T3, T4, T5, T6, Fz, Cz, Pz). Impedances were at or below $5000 \Omega$. A digital electroencephalograph (Medicid 3E, Neuronic Mexicana) with a gain of 10000 was used; amplifier bandwidth was set between 0.3 and $30 \mathrm{~Hz}$, and the sampling rate was $100 \mathrm{~Hz}$. EEG recordings were registered for at least 30 min during spontaneous sleep using monopolar derivations with linked ear lobes as the reference. Simultaneously to EEG, electrocardiogram (EKG), electromyogram (EMG), and electrooculogram (EOG) were recorded (Fig. 1). At the beginning of the second stage of this study, IDA children were supplemented with elemental iron $(5 \mathrm{mg} / \mathrm{kg} /$ day; ferrous fumarate; Ferval-Valdecasas, México) for 4 months, while clinical checkups were carried out every 4 weeks. All tests were repeated after finishing iron supplementation.

\section{Data analysis \\ $\mathrm{Hb}$ and iron profile}

A Mann-Whitney test was applied to assess mean differences previous therapy, IDA vs. CTL, and post treatment, IDA-IS vs. IDA.

\section{qEEG}

For qEEG analysis, 24 artifact-free segments lasting 2.56 seconds taken from stage II of quiet sleep were visually chosen (Fig. 1). The Fast Fourier Transform and cross-spectral matrices were calculated every
$0.39 \mathrm{~Hz}$ for delta $(1.56-3.51 \mathrm{~Hz})$, theta $(3.90$ $7.80 \mathrm{~Hz})$, alpha $(8.19-12.48 \mathrm{~Hz})$, beta $(12.87-$ $19.11 \mathrm{~Hz})$, and total $(1.56-19.11 \mathrm{~Hz})$ frequency bands, for each monopolar channel. The constant scale factor geometric power (GP) ${ }^{23-25}$ was applied to ponder body growth and the changing physical properties with age of the conductive volume. This is, the intrinsic physical features of skull bone and skin, geometry and conductance, which importantly contribute to inter-individual variability. Therefore, each subject's $\mathrm{GP}^{24}$ was subtracted from the individually obtained cross-spectral matrix and then Absolute Power (AP) was determined for each frequency band (delta, theta, alpha, and beta) and electrode or derivation. AP is the area under the curve of the corresponding power spectrum, and corresponding to the EEG energy at the specific frequency interval. From the broad-band spectral values or their $Z$-transform interpolated maps were produced and these compared against previously built norms, created per age and gender in our laboratory. ${ }^{21}$

Absolute power (AP) maps were plotted after subtracting GP. ${ }^{23-25}$ It was determined that the contribution of GP to overall EEG variance reached up to $42 \%$ of corrected data at each age..$^{23,24}$ This correction allowed us to remove non-physiological variability from the analysis, leading to the construction of highly accurate EEG maps. Similarly, relative power (RP) was calculated for each frequency band. This is defined as the AP of the respective band divided by the overall or total AP (i.e. all bands included). Intuitively, RP of each band represents the percentage of power contributed to total energy.

Both AP and RP are necessary for analysis. Though $\mathrm{RP}$ is not sensitive to differences within the same subject, any observed reduction of RP may be a consequence of AP reduction in a specific frequency band or to an increase of AP in the remaining bands. In order to clarify this both RP and AP were calculated and analyzed.

The qEEG of each IDA child was compared with the previously created norms. ${ }^{22}$ The developed qEEG-norms software was applied to plot the differences, in terms of the $Z$ transformation of the original data, between the case studied and the corresponding values of the norm for the given age and gender. $Z$ values were obtained applying the formula: $Z=$ $\left[X_{i}-\mu\right] / \sigma$, where $X$ is the raw value of the $i$ th subject, and $\mu$ and $\sigma$ are, respectively, mean and standard deviation of the normal subjects of the same age as the $i$ th subject. After this, a $Z$ map was built for each individual case.

Because of the multivariate nature of the collected EEG data, a non-parametric multivariate permutation test was applied to investigate the existence of 


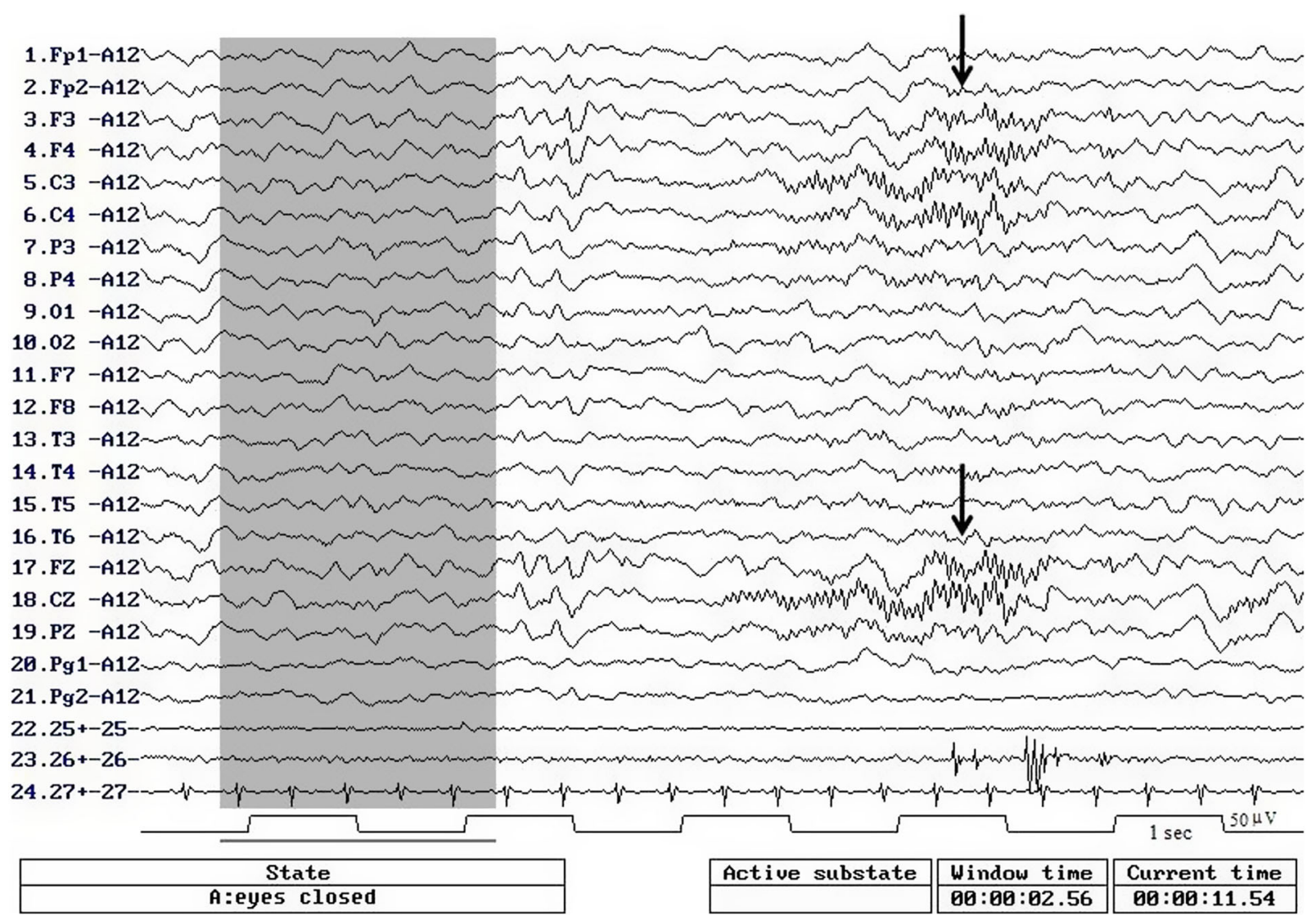

Figure 1 Window analysis of 2.56 seconds (highlighted in gray). EEG (channels 1-19), EOG (channels 20-21), EMG (channels 22-23), and EKG (channel 27). Arrows point to sleep spindles, characteristics of stage II of NREM sleep. All windows were selected where there were no sleep spindles.

differences between groups CTL1 and IDA. The global test was calculated using the permutation distribution of the maximum of Student's $t$-test. After applying the permutation techniques, the estimated distribution for $\max t$ was used to set the significance levels that control and avoid the inflation of type I error. Multiple comparisons were carried out by computing all pairwise comparisons using Student's $t$-test. ${ }^{25}$ Thus, global hypotheses were used to simultaneously test EEG differences for all the leads, and marginal hypotheses were used to test differences per lead. ${ }^{26}$ The AP for each frequency band was independently compared between IDA and CTL1 groups, and before and after iron supplementation (IDA vs. IDA-IS) likewise, it was compared IDA-IS vs. CTL2. In addition, the average AP per group was calculated, and an average map was built; therefore, we obtained average maps for groups CTL1, IDA, and IDA-IS.

In this design, the odds ratio was calculated to measure the effect size of the iron-supplementation treatment.

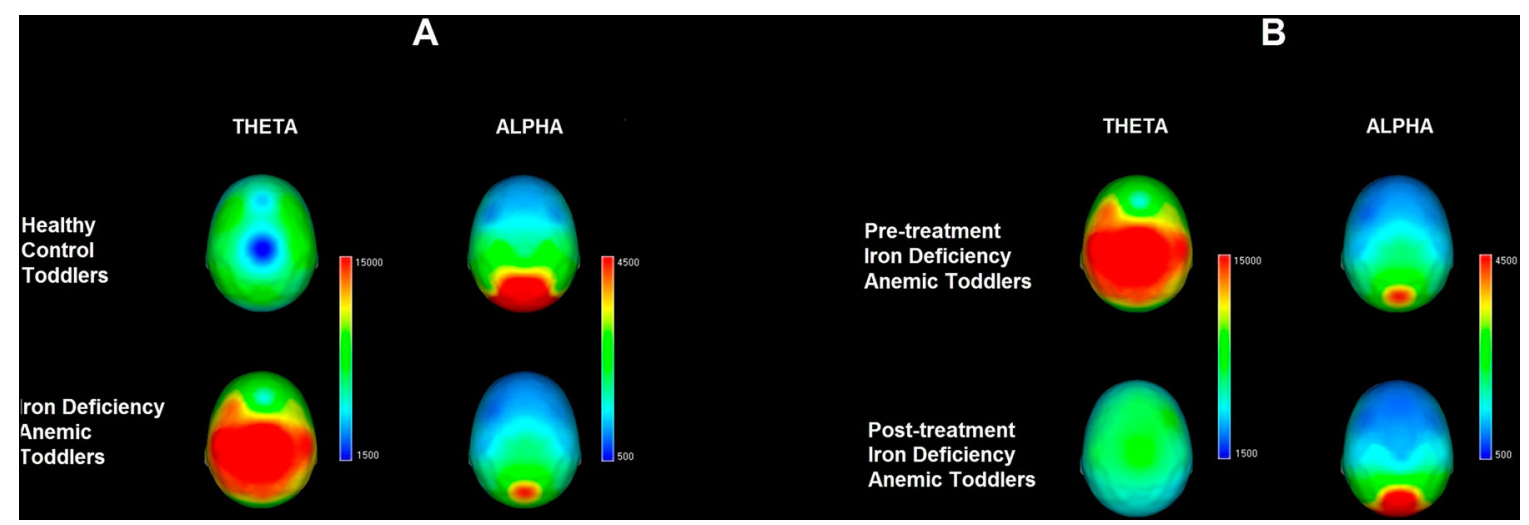

Figure 2 qEEG distribution maps of theta and alpha absolute power. (A) Healthy control infants (CTL1) (top) and iron-deficiency anemic (IDA) infants (bottom). (B) Iron-deficiency anemic infants before (top) and after (bottom) iron supplementation treatment (IDA vs. IDA-IS). 


\section{Results}

\section{First stage}

In Table 1 are shown the mean differences of $\mathrm{Hb}$ and iron profile between IDA and CTL groups. It is evident that IDA group infants present a ferropenic anemia.

When comparing the brain activity maps of IDA children to the previously calculated norms, 16 IDA subjects $(64 \%)$ showed more power in the slower frequencies (i.e. delta and theta) and less power in the alpha band. Figure 2A depicts the average maps of AP for CTL1 and IDA groups.

When applying non-parametric multivariate permutation tests before iron supplementation, we noticed highly significant differences between groups $(P<$ 0.001). In general, theta AP was higher in the IDA group than in the CTL1 group, and alpha AP was higher in centro-posterior areas in the CTL1 group (Fig. 3). On the other hand, delta and theta RP showed significantly higher values in the IDA than in the CTL1 group in fronto-central leads, and alpha $\mathrm{RP}$ was higher in CTL1 than in IDA in centro-posterior regions (Fig. 4).

Figure $5 \mathrm{~A}$ shows an example of qEEG pertaining to an IDA baby and its comparison to the norms. In this figure, a generalized excess of delta AP and RP, applying the GP correction, can be noticed; on the other hand, an excess of theta and a deficit of alpha in relation to the norms is also evinced.

\section{Second stage}

As detailed in the methodology section, IDA infants were supplemented for 4 months with elemental iron under medical supervision (IDA-IS group) to restore their depleted reserves. After the iron-restoration therapy, all studies were repeated and all parameters determined. Hematological values of IDA infants, considered as a group, largely improved (before iron supplementation: mean $=8.2 \pm 0.83 \mathrm{~g} / \mathrm{dl}$; after iron supplementation: $\quad$ mean $=12.4 \pm 1.15 \mathrm{~g} / \mathrm{dl}$ ), and most of them attained figures within normality. Nevertheless, a few cases did not reach values within the limits of normalcy despite the considerable enhancement of bodily iron stores. Therefore, children from IDA-IS group were compared to children from groups IDA and CTL2 to know which changes they had and how normal were the values they reached, respectively.

When IDA group was compared with IDA-IS group, alpha AP increased at occipital, $\mathrm{Cz}$, and $\mathrm{Pz}$ leads while theta AP diminished in almost all leads (Fig. 2B). No significant differences were observed in theta AP between IDA-IS and CTL2, and only alpha $\mathrm{AP}$ at $\mathrm{Cz}$ and $\mathrm{Pz}$ remained without reaching normal values. In relation to RP results, significant delta and theta decreases in fronto-central leads, and significant alpha increases in $\mathrm{C} 3$ and $\mathrm{C} 4$, which reached CTL2 values, were observed. Hence, the gap between IDA and CTL2 infants narrowed after iron supplementation.

Figure 5B shows an example of a baby who reached normal $\mathrm{Hb}$ values after 4 months of iron supplementation. The excess of delta and theta AP disappeared. Alpha AP values were consistently normalized as well.

Before iron-supplementation treatment, 16 out of 25 infants had values different from the normal in their EEGs; after treatment 19 reached normal EEG values. The size effect of the treatment was highly significant (odds ratio $=0.1776,95 \%$ confidence interval: $0.0520-0.6069, Z=2.7570, P=0.0058$ ); odds ratio $<1$ means there is a negative relationship between administration of iron supplement and presence of abnormal values in the EEG.

\section{Discussion}

According to the World Health Organization, ${ }^{20}$ in any society the prevalence of anemia should ideally be less than $5 \%$ and not beyond $19 \%$, a situation defined by WHO as a mild public health issue. Beyond those figures $(20-39.9 \%)$ WHO considers it a moderate problem, and severe if prevalence is greater than $40.0 \%$. In Mexico, according to the 2012 National Survey of Health and Nutrition ${ }^{27}$ (Ensanut 2012), the estimated prevalence of anemia among children younger than 5 years old was $23.3 \%$, while the highest $(38.3 \%)$ was determined within the group of 12-23 months old children. ${ }^{27}$ Those figures according to WHO's criteria set the prevalence of anemia among Mexican infants and toddlers at the scale of a moderate public health issue. Adequate body iron stores are crucial for optimal physical and cognitive development due to the important role this element plays in harnessing metabolic energy. In this order of thoughts, innumerable metabolic events depend on the presence of satisfactory iron body stores. Specifically, brain development results from glial and neuronal differentiation. The latter cells require considerable amounts of metabolic energy for migration, the extension of neuritic processes (axons and dendrites), the establishment of synaptic contacts, and particularly myelination. All these events require a considerable and continuous supply of energy. In this sense and at the long term, anemia is a pathological condition posing an immensely and immediate high risk for the wholesome physical and cognitive development of children suffering from it. Anemic infants are likely to later underachieve academically and not reaching their full adult-life potential. ${ }^{28}$

Anemia in children younger than 5 years old has a particularly negative impact on their psychophysiological development and later school performance, and associated with developmental delays including 

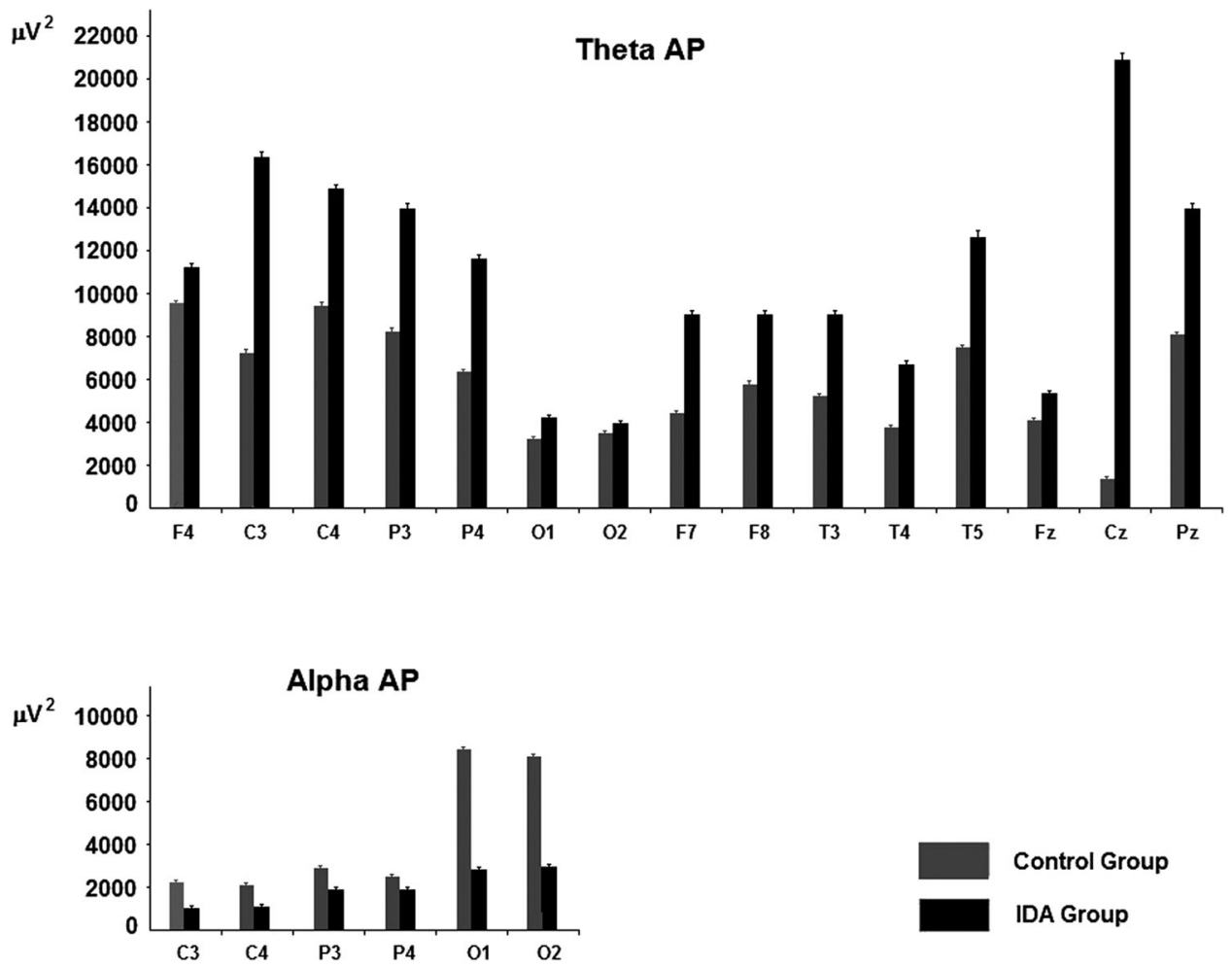

Figure 3 Absolute power means and standard errors of the iron-deficient anemic (IDA) group and the control (CTL1) group. Only leads with significant differences between groups are shown.

poorer abilities in fine and gross motor skills, visualmotor integration, language acquisition, and total IQ. ${ }^{17}$

Here we studied CNS development in a group of anemic infants through qEEG and observed a delayed maturation in terms of persistence of slower activities at the expense of faster ones. In order to determine the occurrence of different extents of susceptibility in relation to nutritional status, i.e. nutritional IDA, individual comparisons of qEEG were made from each IDA infant to a collection of previously calculated qEEG norms. ${ }^{22}$ Initially, it was manifest the presence of more theta AP and less alpha AP in the IDA group compared to group CTL1 (see Fig. 2A). Not all IDA cases, however, showed qEEG activity whereby stronger slower activity (delta and theta powers) was present at the expense of faster one (alpha). Nevertheless, only a minor percentage of IDA infants, 36\%, showed a qEEG developmentally corresponding to their age. This is, most IDA infants, $64 \%$, were determined as significantly stricken by IDA, and showed an underdeveloped qEEG.

CNS maturation observed as the appearance of characteristic frequency qEEG patterns manifests itself as a progressive acquisition and increase of faster waves (alpha and beta) with a corresponding decrease of slower activity (delta and theta). A considerable number of qEEG studies has provided evidence on the observed changes with age. ${ }^{29-33}$ In our study and in contrast to the normally observed developmental pattern, 16 children out of a total of 25 IDA cases showed more power in slow bands and less power in fast activity than that pattern corresponding to their age. Our observations demonstrate a delay in CNS maturation in IDA children. This was confirmed by the multivariate permutation tests analysis (Figs. 3 and 4).

In a previous study ${ }^{34}$ IDA school-age children (810 years old) showed an immature qEEG showing higher delta AP in frontal regions, and also higher theta activity in all derivations compared to their iron replete contemporaries. Thus, the presence of an immature qEEG among IDA infants, children, and pre-teens has been a consistent finding. In this sense, Peirano et al. ${ }^{35}$ found reduced density, lower frequency, and longer inter-spindle intervals of sleep spindles in iron-deficient, anemic, 6-month-old infants during NREM sleep (no rapid eye movements sleep) at stages 2 and 3-4, suggesting an abnormal developmental progression of the NREM sleep pattern. These results support our hypothesis predicting a maturational delay in the CNS development of IDA infants. Few papers report alterations in eventrelated potentials in IDA and iron-deficient nonanemic children. For instance, Monga et al. ${ }^{15}$ found longer absolute peak latencies of visually evoked potentials among IDA babies in relation to their non-anemic contemporaries, results which the authors suggest may be explained by the hypoxia 

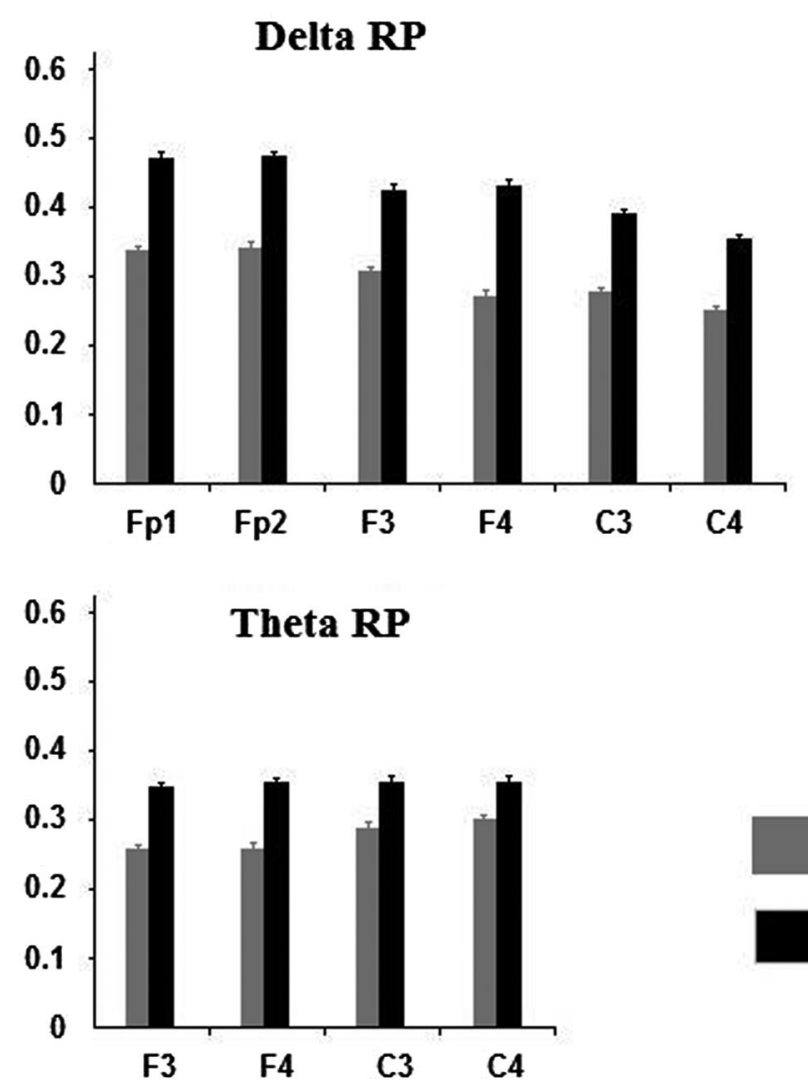

Control Group

\section{IDA Group}

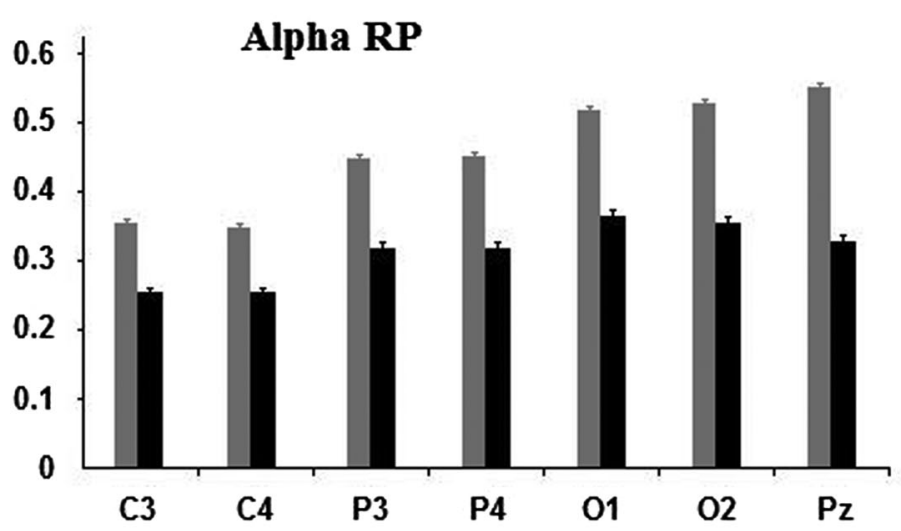

Figure 4 Relative power means and standard errors of the iron-deficient anemic (IDA) group and the control (CTL1) group. Only leads with significant differences between groups are shown.

present together with anemia. Similar results were found by Algarin et al. ${ }^{13}$ In the present study, all tests were repeated after 4 months of iron supplementation to the initially IDA children. After the restoration of body iron stores, qEEG features among IDA-IS infants improved, although not all of
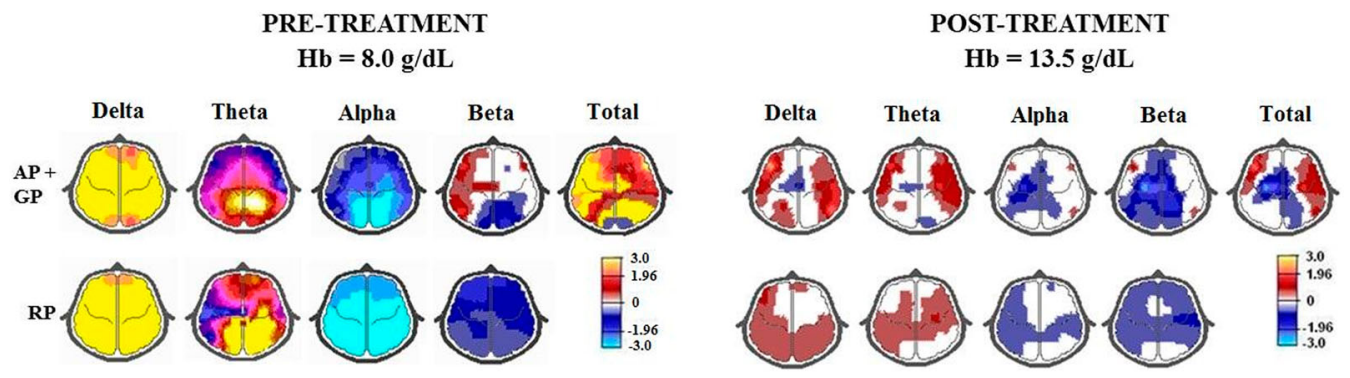

Figure $5 Z$ maps obtained from qEEG of an IDA infant before and after iron restitution treatment. Hb: hemoglobin; AP + GP: absolute power with geometric power correction; RP: relative power. The color coding indicates the magnitude of statistical deviation in the mapped brain areas, from -3 to $+3 Z$ values in relation to the norms. Statistically significant values $( \pm 1.96)$ are indicated in the color coding bar. 
them showed complete qEEG normalization. Likewise, in group IDA-IS the multivariate permutation analysis tests showed an improvement of alpha $\mathrm{AP}$ in leads $\mathrm{O} 1, \mathrm{O} 2, \mathrm{Cz}$, and $\mathrm{Pz}$ as compared to their previous IDA condition. Excess of theta AP disappeared. Nevertheless, alpha AP remained lower compared to control values in middle central and parietal leads. This further comparison was made against another control group, CTL2, of ages between 7 and 16 months matched accordingly to the present age of IDA-IS children. This is, it was taken into account that iron supplementation lasted 4 months and at this stage brain developmental changes occur rapidly.

Kececi and Degirmenci ${ }^{36}$ studied both the qEEG and cognitively evoked related potentials (ERPs) in a sample of adult patients with iron-deficient anemia before and after a three months iron-restoration therapy. After iron therapy, some ERPs latencies decreased while the amplitudes increased. On the other hand, qEEG analysis showed an increase of power in all EEG bands. Otherwise, qEEG analysis showed an increase in power in all EEG bands after iron therapy. The cited authors discuss and show the parallel presence of low hemoglobin levels, consequently of chronic hypoxia and how these two conditions may explain ERPs' alterations both in amplitude and latency as well as the decrease of qEEG power before recovery of normal hematological parameters. That study was conducted among adults, 20-60 years old, and is not directly comparable to our work which was done in babies. Nevertheless, in both situation, it is clear that iron-deficiency anemia is accompanied by alterations in brain electrical activity.

Nevertheless, the extent of the impact of iron supplementation remains under scrutiny. Bandhu et al. ${ }^{37}$ studied a group of school-age children (8-10 years old) who were divided into two subgroups according to serum hemoglobin $(\mathrm{Hb})$ concentration: groups $\mathrm{C}$ $(n=34 ; \mathrm{Hb}>12 \mathrm{~g} / \mathrm{dl})$, and anemic $(n=18 ; \mathrm{Hb}<$ $12 \mathrm{~g} / \mathrm{dL}$ ). Anemic children received iron-restoration therapy for 3 months, and all tests were performed both pre- and post-iron therapy: auditory ERPs were recorded applying an odd ball paradigm, and also psychometric tests were carried out. Pre-treatment, P300 latency was significantly longer among anemic children compared to group C. Psychometric testing also showed better scores attained by $\mathrm{C}$ children. After iron therapy and when hematological parameters reached normal values amongst anemic children, P300 latency decreased. Nevertheless, in spite of 3 months body iron-restoration therapy statistical electrophysiological differences persisted between anemic children pre- and post Fe treatment compared to group C. This is, some underlying neural deficits remained. Posterior to iron therapy the psychometrics scores did reach those attained by group $\mathrm{C}$, indicating an improvement in cognitive performance, this resulting mainly from improved attention and concentration scores. Nevertheless, according to the authors, the persistence of ERPs deficits is indicative of incomplete normalization of brain function.

Previous research demonstrated that ERPs were markedly altered among IDA children, brain activity which normalized after iron supplementation and restoration of normal hematological parameters. ${ }^{38,39}$ Brain development takes place speedily, and when the critical windows of development are surpassed, either attaining or not full capabilities will strongly depend on the individual's nutritional integrity and environmental influences. Restoring not reached capabilities should be a priority of modern research.

In general, most studies ${ }^{9,13,40,41}$ identify long-lasting consequences due to iron-deficiency and irondeficiency anemia present along infancy. Prospective, long-term studies have shown that neurological and cognitive alterations persist in spite of pursuing a therapy of iron restoration. ${ }^{40-42}$ A similar situation has been observed in the neuroendocrine system. ${ }^{43,44}$ All studies cited here denote the potentially negative impact of iron deficiency with or without anemia on neurodevelopment, an impact which appears to be harsher when the metal deficiency is suffered early in life.

In relation to ours and others' studies, the accumulating evidence indicates that iron deficiency is an important neurodevelopmental risk factor, and although restoration of bodily iron stores may significantly reduce the observed neurophysiological alterations, the same available evidence indicates it may not be enough to restore full capabilities. For these reasons among infants iron-deficiency with or without anemia is an important risk factor for CNS development.

Strengths and limitations of the study: Few attempts have been made to assess CNS maturation in IDA infants employing neurophysiological techniques. Through the study of qEEG, this work shows the existence of a delay in CNS development among iron anemic infants. They appear to recover substantially after iron supplementation, and although the qEEG maturational recovery among iron-deficient anemic infants is substantial, it is yet observed incomplete. The partial qEEG recovery could be due to either endogenous CNS repair limitations, the complexity of brain development, and other pre-existent and persistent nutritional deficiencies, as has been pointed out by us and other authors, or more prolonged Fe therapy may be required. Nutritionally the optimal arrival to the opening and closing of critical windows of development is mandatory. Otherwise, normal development is at risk. Nevertheless, the 
magnitude of recovery after iron supplementation therapy among IDA infants in reducing qEEG abnormalities is revealing and highly promising. This is a low cost and simple therapy which importantly contributes to reducing the possible behavioral and cognitive sequelae caused by the deficit of dietary iron. Therefore, iron-deficiency anemia constitutes an important risk factor for CNS development among infants.

\section{Acknowledgments}

The authors thank Jessica González Norris for reviewing English.

\section{Disclaimer statements \\ Contributors None.}

Funding All phases of this study were partially supported by a grant from the National Council of Science and Technology (CONACYT) Project No. CO1/40257-A1.

\section{Conflicts of interest None.}

Ethics approval This research project was pre-approved by the Bioethics Committee of the School of Medicine, UAEMEX and by the participating hospitals: Children's Hospital, IMIEM and General Hospital Adolfo López Mateos, ISEM. Approved in March/ 2014.

\section{ORCID}

Gloria A. Otero (1) http://orcid.org/0000-0003-05481387

Thalia Fernández (1) http:// orcid.org/0000-0002-28427773

F. Bernardo Pliego-Rivero (1) http://orcid.org/00000002-4261-6511

\section{References}

1 Miller JL. Iron deficiency anemia: a common and curable disease. Cold Spring Harb Perspect Med 2013;3(7):a011866.

2 Levin C, Harpaz S, Muklashi I, Lumelsky N, Komisarchik I, Katzap I, et al. Iron deficiency and iron-deficiency anemia in toddlers ages 18 to 36 months: a prospective study. J Pediatr Hematol Oncol 2016;38(3):205-9.

3 McLean E, Cogswell M, Egli I, Wojdyla D, de Benoist B. Worldwide prevalence of anaemia, WHO vitamin and mineral nutrition information system, 1993-2005. Public Health Nutr 2009;12(4):444-54.

4 WHO. Worldwide prevalence of anaemia 1993-2005: WHO global database on anaemia. Geneva, Switzerland: World Health Organization, 2008.

5 Abbaspour N, Hurrell R, Kelishadi R. Review on iron and its importance for human health. J Res Med Sci 2014;19(2):164-74.

6 Domellöf M, Braegger C, Campoy C, Colomb V, Decsi T, Fewtrell $\mathrm{M}$, et al. Iron requirements of infants and toddlers. J Pediatr Gastroenterol Nutr 2014;58(1):119-29.

7 Chandra J. Combating iron deficiency in children. Indian J Pediatr 2013;80(12):983-4.

8 Carter RC, Jacobson JL, Burden MJ, Armony-Sivan R, Dodge $\mathrm{NC}$, Angelilli ML, et al. Iron deficiency anemia and cognitive function in infancy. Pediatrics 2010;126(2):e427-34.
9 Lozoff B, Beard J, Connor J, Felt B, Georgieff M, Schallert T. Long-lasting neural and behavioral effects of iron deficiency in infancy. Nutr Rev 2006;64(5 Pt 2):34---43; discussion S72-S91.

10 McCann JC, Ames BN. An overview of evidence for a causal relation between iron deficiency during development and deficits in cognitive or behavioral function. Am J Clin Nutr 2007;85(4): 931-45.

11 Pettit K, Rowley J, Brown N. Iron deficiency. Paediatr Child Health 2011;21(8):339-43.

12 Sarici SÜ, Serdar MA, Dündaröz MR, Ünay B, Akin R, Deda $\mathrm{G}$, et al. Brainstem auditory-evoked potentials in iron-deficiency anemia. Pediatr Neurol 2001;24(3):205-8.

13 Algarín C, Peirano P, Garrido M, Pizarro F, Lozoff B. Iron deficiency anemia in infancy: long-lasting effects on auditory and visual system functioning. Pediatr Res 2003;53(2):217-23.

14 Burden MJ, Westerlund AJ, Armony-Sivan R, Nelson CA, Jacobson SW, Lozoff B, et al. An event-related potential study of attention and recognition memory in infants with irondeficiency anemia. Pediatrics 2007;120(2):e336-45.

15 Monga M, Walia V, Gandhi A, Chandra J, Sharma S. Effect of iron deficiency anemia on visual evoked potential of growing children. Brain Dev 2010;32(3):213-6.

16 Beard JL, Wiesinger JA, Connor JR. Pre- and post-weaning iron deficiency alters myelination in Sprague-Dawley rats. Dev Neurosci 2003;25(5):308-15.

17 Hare D, Ayton S, Bush A, Lei P. A delicate balance: iron metabolism and disease of the brain. Front Aging Neurosci 2013;5:34.

18 Beard J. Iron deficiency alters brain development and functioning. J Nutr 2003;133(5 Suppl 1):1468S-72S.

19 Unger EL, Paul T, Murray Kolb LE, Felt B, Jones BC, Beard JL. Early iron deficiency alters sensorimotor development and brain monoamines in rats. J Nutr 2007;137(1):118-24.

20 Petsche H., Pockberger H. Rappelsberger P. On the search for the sources of the electroencephalogram. Neuroscience 1984;11 (1): $1-27$.

21 WHO. Haemoglobin concentrations for the diagnosis of anaemia and assessment of severity. Geneva, Switzerland: World Health Organization; 2011. Available from: http:// www.who.int/vmnis/indicators/haemoglobin/en/

22 Otero GA, Harmony T, Pliego-Rivero FB, Ricardo-Garcell J, Bosch-Bayard J, Porcayo-Mercado R, et al. QEEG norms for the first year of life. Early Hum Dev 2011;87(10):691-703.

23 Valdés-Sosa P, Bosch J, Grave R, Hernández J, Riera J, Pascual $\mathrm{R}$, et al. Frequency domain models of the EEG. Brain Topogr 1992;4(4):309-19.

24 Hernández JL, Valdés P, Biscay R, Virues T, Szava S, Bosch J, et al. A global scale factor in brain topography. Int $\mathbf{J}$ Neurosci 1994;76(3-4):267---78.

25 Szava S, Valdes P, Biscay R, Galan L, Bosch J, Clark I, et al. High resolution quantitative EEG analysis. Brain Topogr 1994;6(3):211-9.

26 Galán L, Biscay R, Rodríguez JL, Pérez-Abalo MC, Rodríguez $\mathrm{R}$. Testing topographic differences between event related brain potentials by using non-parametric combinations of permutation tests. Electroenceph Clin Neurophysiol 1997;102(3):240-7.

27 National Nutrition Survey. 2006. Available from: http://www. insp.mx/ensanut2006.pdf

28 Estrada JA, Contreras I, Pliego-Rivero FB, Otero GA. Molecular mechanisms of cognitive impairment in iron deficiency: alterations in brain-derived neurotrophic factor and insulin-like growth factor expression and function in the central nervous system. Nutr Neurosci 2014;17(5):193-206.

29 Matousek M y Petersen I. Frequency analysis of the EEG in normal children and adolescents. In: Kellaway P, Petersen I, (eds.) Automation of clinical electroencephalography. New York: Raven Press, 1973, p. 75---102.

30 Hagne I, Persson J, Magnusson R, Petersen I. Spectral analysis via fast Fourier transform of waking EEG in normal infants. In: Kellaway P, Petersen I, (eds.) Automation of clinical electroencephalography. New York: Raven Press; 1973. p. 103---43.

31 John E.R., Ahn H., Prichep L., Trepetin M., Brown D., Kaye H. Developmental equations for the electroencephalogram. Science 1980;210(4475):1255-8.

32 Harmony T, Marosi E, Díaz de León AE, Becker J, Fernández T. Effect of sex, psychosocial disadvantages and biological risk factors on EEG maturation. Electroenceph Clin Neurophysiol 1990;75(6):482-91.

33 Clarke AR, Barry RJ, McCarthy R, Selikowitz M. Age and sex effects in the EEG development of the normal child. Clin Neurophysiol 2001;112(5):806-14. 
34 Otero GA, Aguirre DM, Porcayo R, Fernández T. Psychological and electroencephalographic study in school children with iron deficiency. Int J Neurosci 1999;99(1-4):113-21.

35 Peirano P, Algarín C, Garrido M, Algarín D, Lozoff B. Irondeficiency anemia is associated with altered characteristics of sleep spindles in NREM sleep in infancy. Neurochem Res 2007;32(10):1665-72.

36 Kececi H, Degirmenci Y. Quantitative EEG and cognitive evoked potentials in anemia. Neurophysiol Clin 2008;38(2):137-43.

37 Bandhu R, Shankar N, Tandon OP, Madan N. Effects of iron therapy on cognition in anemic school going boys. Indian $\mathbf{J}$ Physiol Pharmacol 2003;47(3):301-10.

38 Otero GA, Pliego-Rivero FB, Contreras G, Ricardo J, Fernández T. Iron supplementation brings up a lacking P300 in iron deficient children. Clin Neurophysiol 2004;115(10):2259-66.

39 Otero GA, Pliego-Rivero FB, Porcayo-Mercado R, MendietaAlcántara G. Working memory impairment and recovery in iron deficient children. Clin Neurophysiol 2008;119(8):1739-46.
40 Lukowski AF, Koss M, Burden MJ, Jonides J, Nelson CA, Kaciroti N, et al. Iron deficiency in infancy and neurocognitive functioning at 19 years: evidence of long-term deficits in executive function and recognition memory. Nutr Neurosci 2010;13 (2):54-70.

41 Lozoff B. Early iron deficiency has brain and behavior effects consistent with dopaminergic dysfunction. J Nutr 2011;141(4): $740 \mathrm{~S}-6 \mathrm{~S}$.

42 Algarín C, Nelson CA, Peirano P, Westerlund A, Reyes S, Lozoff B. Iron-deficiency anemia in infancy and poorer cognitive inhibitory control at age 10 years. Dev Med Child Neurol 2013;55(5): 453-8.

43 Felt B, Jimenez E, Smith J, Calatroni A, Kaciroti N, Wheatcroft $\mathrm{G}$, et al. Iron deficiency in infancy predicts altered serum prolactin response 10 years later. Pediatr Res 2006;60(5):513-7.

44 Felt BT, Peirano P, Algarín C, Chamorro R, Sir T, Kaciroti N, Lozoff B. Long-term neuroendocrine effects of iron-deficiency anemia in infancy. Pediatr Res 2012;71(6):707-12. 